\title{
Processing doubly quantified sentences: Evidence from eye movements
}

\author{
RUTH FILIK \\ University of Glasgow, Glasgow, Scotland \\ KEVIN B. PATERSON \\ University of Leicester, Leicester, England \\ and \\ SIMON P. LIVERSEDGE \\ University of Durham, Durham, England
}

\begin{abstract}
We investigated the processing of doubly quantified sentences, such as Kelly showed a photo to every critic, that are ambiguous as to whether the indefinite (a photo) specifies single or multiple referents. Ambiguity resolution requires the computation of relative quantifier scope: Whether a or every takes wide scope, thereby determining how many entities or events are to be represented. In an eye-tracking experiment, we manipulated quantifier order and whether continuations were singular or plural, for constructions with the direct or the indirect object occurring first. We obtained effects consistent with the on-line processing of relative scope at the doubly quantified phrase and considered two possible explanations for a preference for singular continuations to the quantified sentence. We conclude that relative quantifier scope is computed on line during reading but may not be a prerequisite for the resolution of definite anaphors, unless required by secondary tasks.
\end{abstract}

Since the 1960 s, research in which sentence processing has been investigated has concentrated on parsing: the process of assigning syntactic structure to a sentence. In recent work, there has been increasing emphasis on the real-time interpretation of linguistic input (e.g., Frazier, Pacht, \& Rayner, 1999). During sentence interpretation, semantic and pragmatic factors are considered along with individual word meanings and knowledge about grammatical structure to produce a representation of sentence meaning as a whole. In constructing this representation, it is important to establish how many entities or events are being described. Quantifiers (e.g., some, all) provide this information.

Sentences with two or more different quantifiers may be ambiguous, since each can indicate a different number of entities or events. Consider Sentence 1, containing $a$ and every:

(1) Kelly showed a photo to every critic.

The indefinite $a$ indicates that there is at least one instance of an entity (i.e., one photo). The universal quan-

This research was supported by an Experimental Psychology Society Study Visit Grant and Grant S19168 from the Biotechnology and Biological Sciences Research Council (U.K.). A complete set of experimental materials may be obtained by e-mail from the first author. We are grateful to Keith Rayner and an anonymous reviewer for helpful reviews. Correspondence concerning this article should be addressed to R. Filik, Department of Psychology, University of Glasgow, 58 Hillhead Street, Glasgow G12 8QB, Scotland (e-mail: r.filik@gla.ac.uk). tifier every cues the instantiation of multiple entities or events. Unambiguous sentence interpretation depends on the reader's establishing relative quantifier scope, with one quantifier taking wide scope over the other, thereby determining how many entities or events are represented. For Sentence 1, every must take scope over the indirect object (IO); conventionally, it is understood that there is more than one critic. However, the number of photos remains ambiguous. If $a$ takes wide scope, one photo will be represented, but if every takes wide scope, the sentence may be interpreted as stating that Kelly showed each critic a different photo. Alternatively, it may be interpreted as stating that the same photo was shown to a different critic on separate occasions (see Poesio, 1996). However, it generally is assumed that the nonspecific reading, with the indefinite quantifier referring to many entities, is to be preferred over the one with its referring to a single specific entity (Fodor \& Sag, 1982).

We investigated three hypotheses concerning relative quantifier scope. The linear order principle requires that the first quantifier take wide scope (Johnson-Laird, 1969; Lakoff, 1971). By contrast, Ioup (1975) argued that quantifier characteristics and the grammatical function of quantified phrases interact to determine scope preferences. Ioup proposed a quantifier hierarchy and a grammatical function hierarchy. For a sentence with two quantifiers, the quantifier hierarchy principle requires that the quantifier highest in the hierarchy take wide scope ( $>$ indicates takes scope over):

each $>$ every $>$ all $>$ most $>$ many $>$ several $>$ some $>$ a few . 
Ioup did not include $a$ within her hierarchy but suggested that it falls between every and all. The grammatical hierarchy principle requires that a quantified phrase in one syntactic position take scope over one in a position lower on the hierarchy:

topic $>$ deep and surface subject $>$ deep subject or surface subject $>$ indirect object $>$ prepositional object $>$ direct object.

Fodor (1982) suggested that linear order and quantifier characteristics interact. When every precedes $a$, both linear order and quantifier characteristics favor an interpretation in which the indefinite quantifier refers to multiple entities. However, when a precedes every, linear order favors an interpretation in which the indefinite quantifier refers to one entity but the characteristics of every favor an interpretation in which the indefinite quantifier refers to many. Fodor argued that in the latter case, readers initially build a representation in which the indefinite quantifier refers to one entity and, on encountering every, revise it to include multiple entities.

Despite the interest in factors likely to affect scope, there has been limited empirical investigation of their influence on comprehension. Initial studies (Catlin \& Micham, 1975; Ioup, 1975; Johnson-Laird, 1969; Micham, Catlin, VanDerveer, \& Loveland, 1980) used judgments concerning the number of entities or events involved in a situation and are not necessarily informative about on-line sentence interpretation. Kurtzman and MacDonald (1993) used a task that was more sensitive to initial comprehension. They examined participants' judgments about the acceptability of continuations to ambiguous sentences and found high consistency in judgments for some sentences, but not for others. They could not uniquely identify the principles involved in scope resolution and, instead, argued that their results depended on the interaction of multiple principles. They argued that when principles collectively favor one representation, that representation is built, but if principles are in conflict, competition between the alternative representations occurs before one is selected. Competition-based accounts of parsing predict greater processing difficulty at an ambiguity when there is competition between alternative analyses than when one analysis is strongly favored (e.g., van Gompel, Pickering, \& Traxler, 2000). If competition guides scope ambiguity resolution, we might expect processing difficulty when principles are in conflict.

In two unpublished studies, on-line scope processing has been examined. Tunstall (1998) examined grammaticality decision latencies for continuations to ambiguous sentences, such as The photo(s) was/were of a run-down building as a continuation to Sentence 1. Latencies were shorter for singular than for plural continuations for the $a$-every quantifier order but were the same for every-a. Tunstall argued that participants assigned a wide scope to the first quantifier, instantiating a single entity when it was indefinite, but underspecifying when it was every. However, tasks involving grammaticality judgments might not exclusively reflect normal comprehension processes.
Gillen (1991) examined sentence-by-sentence self-paced reading times and found that singular continuations were read more quickly than plurals regardless of relative quantifier order. She argued that during normal reading, participants preferentially instantiate a singular referent for an indefinite quantifier and adopt more complex representations only if the task includes explicit number judgments.

We employed eye tracking to investigate normal reading. This methodology provides an indication of the moment-to-moment processes in reading (Liversedge \& Findlay, 2000; Rayner, 1998). We manipulated linear order ( $a$ or every first), grammatical order (direct object [DO] or IO first), and noun phrase (NP) anaphor continuation (singular or plural). Anaphors always referred to the indefinite quantifier. We examined three hypotheses concerning which interpretation will be assigned to an ambiguity and two hypotheses concerning how it will be processed (see Table 1).

Effects at the NP anaphor should be informative about how the ambiguity is interpreted. The linear order principle predicts that the first quantifier takes wide scope; when the indefinite quantifier is first, it is assigned a singular interpretation, whereas when every is first, the indefinite quantifier is assigned a plural interpretation. Consequently, reading times should be shorter for singular than for plural NP anaphors with the indefinite quantifier first and shorter for plurals with every first. The grammatical hierarchy principle predicts that the quantifier associated with the IO takes wide scope. Thus, reading times for sentences with the DO first should be shorter for plurals following $a$-every and shorter for singulars following every-a. Conversely, reading times for sentences with the IO first should be shorter for singulars following $a$-every and for plurals following every- $a$. Linear order and grammatical hierarchy principles make the same predictions for IO-first sentences but opposing predictions for DO-first sentences. The quantifier hierarchy principle requires that every take wide scope, with the indefinite quantifier assigned a plural interpretation. It predicts shorter reading times for plural NP anaphors, regardless of the grammatical position of quantifiers or their relative order.

In previous studies, effects at a continuation have been examined. If relative scope is computed on line, we might obtain effects at the quantified region too. Recall that Ioup (1975) considered that scope resolution depends on an interaction involving quantifier and grammatical hierarchies and that Kurtzman and MacDonald (1993) argued that an interaction between principles should incur a processing cost if they impose conflicting demands. Thus, we might expect reading difficulty when quantifier and grammatical hierarchy principles favor alternative scope analyses, with longer reading times for every-a than for $a$-every DO-first sentences and for $a$-every than for every- $a$ IO-first sentences. Finally, Fodor (1982) proposed that an interaction of linear order and quantifier characteristics would produce reading difficulty when the indefinite quantifier is first. 
Table 1

Predictions Concerning Reading Time Effects for Plural (PI) and Singular (S) Noun Phrases at the NP Anaphor Region, Including Examples of DO-First and IO-First Sentences Used in the Experiment

\begin{tabular}{|c|c|c|c|c|}
\hline Condition & & $\begin{array}{c}\text { Linear } \\
\text { Order } \\
\text { Principle }\end{array}$ & $\begin{array}{c}\text { Grammatical } \\
\text { Hierarchy } \\
\text { Principle }\end{array}$ & $\begin{array}{c}\text { Quantifier } \\
\text { Hierarchy } \\
\text { Principle }\end{array}$ \\
\hline \multirow[t]{2}{*}{ A } & DO-first / a-every & & & \\
\hline & $\begin{array}{l}\text { The celebrity gave }{ }_{1} \mid \text { an in depth interview to every reporter from the } \\
\text { newspaper, but }{ }_{2} \mid \text { the interview(s) was/were }\left.\right|_{3} \mid \text { not very }{ }_{4} \mid \text { interesting. }\end{array}$ & $\mathrm{S}<\mathrm{Pl}$ & $\mathrm{Pl}<\mathrm{S}$ & $\mathrm{Pl}<\mathrm{S}$ \\
\hline \multirow[t]{2}{*}{ B } & DO-first / every- $a$ & & & \\
\hline & $\begin{array}{l}\text { The celebrity gave } \mid \text { every in depth interview to a reporter from the } \\
\text { newspaper, but } t_{2} \mid \text { the reporter(s) was/were }{ }_{3} \mid \text { not very }{ }_{4} \mid \text { interested. }_{5}\end{array}$ & $\mathrm{Pl}<\mathrm{S}$ & $\mathrm{S}<\mathrm{Pl}$ & $\mathrm{Pl}<\mathrm{S}$ \\
\hline \multirow[t]{2}{*}{$\mathrm{C}$} & IO-first / $a$-every & & & \\
\hline & $\begin{array}{l}\text { The celebrity gave }{ }_{1} \mid \text { a reporter from the newspaper every in depth interview, } \\
\text { but }_{2} \mid \text { the reporter(s) was } / \text { were }_{3} \mid \text { not very }{ }_{4} \mid \text { interested. }_{5}\end{array}$ & $\mathrm{~S}<\mathrm{Pl}$ & $\mathrm{S}<\mathrm{Pl}$ & $\mathrm{Pl}<\mathrm{S}$ \\
\hline $\mathrm{D}$ & $\begin{array}{l}\text { IO-first / every- } a \\
\text { The celebrity gave }{ }_{1} \mid \text { every reporter from the newspaper an in depth interview, } \\
\text { but }_{2} \mid \text { the interview(s) was/were }{ }_{3} \mid \text { not very }{ }_{4} \mid \text { interesting. } \\
.5\end{array}$ & $\mathrm{Pl}<\mathrm{S}$ & $\mathrm{Pl}<\mathrm{S}$ & $\mathrm{Pl}<\mathrm{S}$ \\
\hline
\end{tabular}

Predictions concerning reading time effects for the doubly quantified region:

Ioup (1975)

Grammatical hierarchy $\times$ quantifier hierarchy

Fodor (1982)

Linear order $\times$ quantifier characteristics 'every interview . . . to a reporter' $>$ 'an interview. . . to every reporter' 'a reporter . . . every interview' $>$ 'every reporter . . . an interview'

'an interview. . . to every reporter' $>$ 'every interview . . . to a reporter' 'a reporter . . . every interview' $>$ 'every reporter . . . an interview'

Note-Vertical lines delimit analysis regions, and slashes denote alternatives.

In addition to these hypotheses, we considered Gillen's (1991) claim that relative scope may not be computed unless the task requires it. If so, we would expect a preference for instantiating singular referents for indefinite quantifiers, with no effects of quantifier or grammatical order, but a processing cost for plural continuations. Finally, DO-first constructions have canonical word order in English, whereas IO-first sentences do not (e.g., Larson, 1988). Perhaps readers would incur a syntactic processing cost for noncanonical IO-first sentences, as compared with canonical DO-first sentences.

\section{METHOD}

\section{Participants}

Fifty-six native English speakers with normal vision from the University of Durham participated.

\section{Materials and Design}

We constructed 48 DO-first or IO-first sentences with an indefinite NP or one quantified by every first (see Table 1). Continuations were singular or plural. Anaphors always referred to the indefinite quantifier. There were eight lists with six items in each form. No item appeared more than once in any list.

We conducted an off-line judgment task to assess how sentences were interpreted. Twenty participants indicated how likely it was that indefinite quantifiers had a plural referent for items without a continuation (e.g., The celebrity gave an in-depth interview to every reporter from the newspaper), using a 5-point scale (1 indicated definitely one, 5 indicated definitely more than one). Table 2 shows the mean plurality ratings.

We conducted 2 (quantifier order) $\times 2$ (grammatical order) analyses of variance (ANOVAs) for participants $\left(F_{1}\right)$ and items $\left(F_{2}\right)$, with effects considered significant when both analyses were reliable $(p<.05)$. Plurality ratings were higher for every - $a$ than $a$-every $\left[F_{1}(1,19)=19.01 ; F_{2}(1,47)=8.94\right]$, consistent with linear order favoring a singular referent when the indefinite quantifier was first and a plural when it was second. Grammatical order was unreliable
$\left[F_{1}<3 ; F_{2}(1,47)=4.98\right]$. The interaction $\left[F_{2}(1,19)=60.53\right.$; $\left.F_{2}(1,47)=107.75\right]$ was consistent with the conjoint operation of quantifier and grammatical hierarchy principles, with higher ratings for $a$-every than for every- $a$ DO-first items $\left[F_{1}(1,19)=37.19\right.$; $\left.F_{2}(1,47)=43.15\right]$ and for every $-a$ than for $a$-every IO-first items $\left[F_{1}(1,19)=62.69 ; F_{2}(1,47)=108.61\right]$. Overall plurality ratings were low, indicating a weak preference for interpreting the indefinite quantifier as a singular. Nevertheless, our manipulation modulated number judgments, with evidence for linear order effects and the operation of quantifier and grammatical hierarchy principles. We reasoned that eye tracking would enable us to determine whether these factors affected on-line sentence processing in the absence of a judgment task.

We ruled out a potential confound due to differences in the plausibility of plural interpretations of nouns from the ambiguous region (e.g., interview and reporter). Seven raters provided plausibility judgments on a 7-point scale for DO-first sentences with indefinite DOs or IOs that were disambiguated as plurals by including the word different (e.g., a different interview vs. a different reporter), with no difference $[\mathrm{DO}=4.1, S D=1.1$; $\mathrm{IO}=4.0, S D=0.9$; $t(47)<1]$. We also established that the frequency of usage of singular and plural nouns used as anaphors did not differ, using the CELEX database [Baayen, Piepenbrock, \& Gulikers, 1995; singular, 81.8 words/million, $S D=132.3$; plural, 67.2 words $/$ million, $S D=$ $125.2 ; t(47)<1]$.

Experimental lists were displayed in fixed random order, together with 64 items from an unrelated experiment. Target sentences were double-spaced across two lines, with the anaphor cen-

Table 2

Mean Ratings (With Standard Deviations) of Plurality of Indefinite Quantifiers in DO-First and IO-First Sentences With a-every and every-a Order

\begin{tabular}{cccccc}
\hline & \multicolumn{3}{c}{ Sentence } \\
\cline { 2 - 3 } \cline { 5 - 6 } Quantifier Order & \multicolumn{2}{c}{ DO First } & & \multicolumn{2}{c}{ IO First } \\
\cline { 2 - 3 } \cline { 5 - 6 } a-every & 3.1 & 0.5 & & 1.6 & 0.5 \\
every $a$ & 2.1 & 0.6 & & 3.3 & 0.7 \\
\hline
\end{tabular}


tered on line two. The experiment was run in two blocks, with practice items beginning each.

\section{Procedure}

A Fourward Technologies DPI Generation 5.5 eye tracker with an angular resolution of $10 \mathrm{~min}$ of arc monitored gaze location and participants' right-eye movements. A PC displayed materials on a monitor $80 \mathrm{~cm}$ from the participants' eyes. Gaze location was monitored every millisecond. Output was sampled to produce a sequence of fixations, recorded as $x$ and $y$ character positions, with start and finish times.

The participants were instructed to read normally and for comprehension. Once seated at the eye tracker, they completed a calibration procedure. Head movements were minimized with a bite bar. Once the participants finished reading each item, they pressed a key, and a comprehension question was displayed. The participants responded by pressing "yes" or "no" keys, with feedback.

\section{RESULTS}

\section{Regions}

Sentences were divided into analysis regions (see Table 1). Reading times are reported for Regions 2-4. Region 2 comprised the quantified phrases and the conjunction. Region 3 was the NP anaphor. Region 4 contained the words not very.

\section{Analysis}

Short contiguous fixations were pooled automatically. Fixations under $80 \mathrm{msec}$ were incorporated into larger adjacent fixations within one character. Fixations under $40 \mathrm{msec}$ and not within three characters of another fixation were deleted. Before analyzing the data, trials were removed when two or more adjacent regions had zero first-pass reading times, accounting for $1.2 \%$ of the data. Comprehension was high $(96 \%$ correct responses to questions with no significant differences across conditions; $\left.F_{\mathrm{s}}<2.7\right)$.

We computed first-pass and total reading times for each region and second-pass reading times for Region 2. Total reading time summed the duration of all fixations made in a region until the participant pressed a button to indicate that he or she had completed reading the sentence. First- and second-pass reading times enabled us to examine the time course of effects. First-pass reading time summed the duration of fixations made on first entering a region until exiting it and was informative about initial processing. Second-pass reading time was calculated by subtracting first-pass from total reading time for a region and indexed reinspection time. Singular and plural anaphors differed in length by up to 4 characters (mean $=$ 2.1). To correct for length differences, we calculated residual (deviations from predicted scores) first-pass and total reading times for Region 3 (Ferreira \& Clifton, 1986). Residual and raw reading times showed the same statistical effects. Consequently, only analyses for raw times are reported. The data for each region were subjected to 2 (quantifier order) $\times 2$ (grammatical order) $\times$ 2 (continuation number) ANOVAs for participants $\left(F_{1}\right)$ and items $\left(F_{2}\right)$, with effects considered significant when both analyses were reliable $(p<.05)$. Table 3 shows mean reading times for Regions 2-4.

\section{Region 2 (Quantified Region)}

Following Ioup (1975), we predicted that an interaction of quantifier and grammatical hierarchy principles would

Table 3

Mean First-Pass and Total Reading Times (in Milliseconds) for Regions 2-4, Second-Pass Reading Times for Region 2, and Residual First-Pass and Total Reading Times for Region 3 of $a$-every and every-a DO-First and IO-First Sentences With Singular and Plural Continuations

\begin{tabular}{|c|c|c|c|c|c|c|c|c|c|}
\hline \multirow[b]{3}{*}{ Region } & \multirow[b]{3}{*}{ Measure } & \multicolumn{4}{|c|}{$a$-every } & \multicolumn{4}{|c|}{ every-a } \\
\hline & & \multicolumn{2}{|c|}{ Singular } & \multicolumn{2}{|c|}{ Plural } & \multicolumn{2}{|c|}{ Singular } & \multicolumn{2}{|c|}{ Plural } \\
\hline & & $M$ & $S D$ & $M$ & $S D$ & $M$ & $S D$ & $M$ & $S D$ \\
\hline \multicolumn{10}{|l|}{ DO-first } \\
\hline \multirow[t]{3}{*}{2} & First-pass time & 1,931 & 452.2 & 1,787 & 518.0 & 2,011 & 569.7 & 2,059 & 545.4 \\
\hline & Second-pass time & 599 & 665.6 & 755 & 835.2 & 789 & 827.9 & 699 & 869.0 \\
\hline & Total time & 2,530 & 755.1 & 2,541 & 968.9 & 2,800 & 975.4 & 2,759 & 973.3 \\
\hline \multirow[t]{4}{*}{3} & First-pass time & 394 & 110.7 & 455 & 157.7 & 361 & 93.8 & 415 & 131.3 \\
\hline & Residual first-pass time & -194 & 100.1 & -145 & 121.0 & -231 & 95.7 & -194 & 99.3 \\
\hline & Total time & 512 & 204.3 & 605 & 360.2 & 514 & 221.8 & 593 & 292.2 \\
\hline & Residual total time & -272 & 160.9 & -173 & 190.5 & -270 & 154.3 & -202 & 148.2 \\
\hline \multirow[t]{2}{*}{4} & First-pass time & 299 & 78.2 & 285 & 66.7 & 307 & 74.7 & 297 & 73.6 \\
\hline & Total time & 451 & 155.0 & 431 & 124.8 & 450 & 118.0 & 465 & 154.9 \\
\hline \multicolumn{10}{|l|}{ IO-first } \\
\hline \multirow[t]{3}{*}{2} & First-pass time & 1,840 & 474.5 & 1,867 & 555.2 & 1,794 & 438.8 & 1,747 & 516.4 \\
\hline & Second-pass time & 998 & $1,172.3$ & 1,093 & $1,002.2$ & 630 & 702.1 & 689 & 816.3 \\
\hline & Total time & 2,839 & $1,271.2$ & 2,960 & $1,112.1$ & 2,424 & 919.3 & 2,436 & 935.5 \\
\hline \multirow[t]{4}{*}{3} & First-pass time & 368 & 102.0 & 406 & 120.1 & 357 & 93.7 & 453 & 126.2 \\
\hline & Residual first-pass time & -220 & 78.1 & -191 & 95.0 & -232 & 108.7 & -146 & 99.3 \\
\hline & Total time & 552 & 369.8 & 625 & 269.8 & 499 & 248.0 & 621 & 336.7 \\
\hline & Residual total time & -217 & 201.7 & -152 & 184.8 & -286 & 149.5 & -165 & 174.7 \\
\hline \multirow[t]{2}{*}{4} & First-pass time & 283 & 70.8 & 294 & 61.6 & 313 & 91.5 & 299 & 71.4 \\
\hline & Total time & 465 & 180.9 & 446 & 131.5 & 481 & 172.1 & 459 & 134.6 \\
\hline
\end{tabular}


cause reading difficulty when the IO was indefinite, whereas Fodor (1982) predicted that an interaction of linear order and quantifier characteristics would cause difficulty when $a$ preceded every. Gillen (1991) proposed that relative scope is not computed unless the task requires it, in which case no effects should occur at this region.

Total reading times were informative about comprehension difficulty at this region. Reading times were longer for $a$-every than for every- $a$ [2,718 vs. $2,605 \mathrm{msec}$; $\left.F_{1}(1,55)=8.99 ; F_{2}(1,47)=5.79\right]$, with no other main effects $(F \mathrm{~S}<1)$. Readers experienced difficulty when $a$ preceded every, consistent with Fodor's predictions concerning the interaction of linear order and quantifier characteristics. Quantifier and grammatical order interacted $\left[F_{1}(1,55)=72.17 ; F_{2}(1,47)=57.18\right]$, with longer reading times for every $-a$ than for $a$-every DO-first sentences $\left[2,780\right.$ vs. $2,536 \mathrm{msec} ; F_{1}(1,55)=16.72 ; F_{2}(1,47)=$ 11.06], and for $a$-every than for every- $a$ IO-first sentences $\left[2,900\right.$ vs. $2,430 \mathrm{msec} ; F_{1}(1,55)=78.51 ; F_{2}(1,47)=$ 53.26]. The effect was consistent with readers incurring a processing cost when quantifier and grammatical hierarchy principles conflicted. No other interactions were significant $\left(F_{\mathrm{S}}<2.3\right)$. Thus, total times matched with Fodor's (1982) predictions and those derived from Ioup (1975). Contrary to Gillen (1991), the results showed that relative scope is computed during normal reading.

If the participants were immediately sensitive to factors affecting relative scope, effects obtained for the quantified region should be observable in first-pass reading times. Reading times were longer for DO-first than for IO-first sentences $\left[1,947\right.$ vs. $1,812 \mathrm{msec} ; F_{1}(1,55)=16.86$; $\left.F_{2}(1,47)=11.86\right]$, with no other significant main effects $\left(F_{\mathrm{s}}<2.4\right)$. Quantifier and grammatical order interacted $\left[F_{1}(1,55)=14.32 ; F_{2}(1,47)=13.03\right]$, with longer DOfirst reading times for every- $a$ than for $a$-every $[2,035$ vs. $\left.1,859 \mathrm{msec} ; F_{1}(1,55)=11.26 ; F_{2}(1,47)=12.61\right]$. IO-first times did not differ reliably $\left[F_{1}(1,55)=4.96\right.$; $F_{2}<2.5$; a-every, $1,854 \mathrm{msec}$; every-a, $\left.1,771 \mathrm{msec}\right]$. No other interactions were reliable $(F \mathrm{~s}<3.5)$. First-pass effects occurred for DO-first sentences only, with longer reading times when every was first, matching total time effects under these conditions.

By contrast, second-pass times were longer for IO-first than for DO-first sentences [ $853 \mathrm{vs.} 710 \mathrm{msec} ; F_{1}(1,55)=$ $\left.6.37 ; F_{2}(1,47)=4.30\right]$. Reading times also were longer for $a$-every than for every $-a$ [861 vs. $712 \mathrm{msec} ; F_{1}(1,55)=$ $\left.24.06 ; F_{2}(1,47)=11.52\right]$. Quantifier and grammatical order interacted $\left[F_{1}(1,55)=26.36 ; F_{2}(1,47)=26.69\right]$, with longer IO-first reading times for $a$-every than for every- $a\left[1,046\right.$ vs. $660 \mathrm{msec} ; F_{1}(1,55)=29.74 ; F_{2}(1,47)=$ 39.69], but no significant difference for DO-first sentences (a-every, $677 \mathrm{msec}$; every- $a, 744 \mathrm{msec} ; F_{\mathrm{s}}<2.1$ ). The quantifier order and number interaction was unreliable $\left[F_{1}(1,55)=5.54 ; F_{2}<3.1\right]$, with no other effects $\left(F_{\mathrm{S}}<\right.$ 1.7). Thus, second-pass effects occurred for IO-first constructions only, with longer reading times when the indefinite quantifier was first, matching total time effects for these items. The computation of relative quan- tifier scope was delayed for IO-first sentences, probably because their noncanonical structure incurred an additional syntactic processing load during the first pass.

\section{Region 3 (NP Anaphor)}

Effects at the NP anaphor were expected to be informative about how the ambiguity was interpreted, with readers incurring a processing cost when the anaphor mismatched the interpretation given to the indefinite quantifier. The linear order principle predicted that the first quantifier takes wide scope, causing readers to adopt a singular interpretation of the indefinite quantifier when it was first and a plural interpretation of the indefinite quantifier when every was first. The quantifier hierarchy principle predicted that every takes wide scope, with the indefinite quantifier assigned a plural interpretation. The grammatical hierarchy principle predicted that IOs take wide scope, with readers adopting a singular interpretation of indefinite IOs and adopting a plural interpretation of the indefinite quantifier when every quantified the IO.

Effects obtained at the quantified region allowed us to discount Gillen's (1991) claim that relative quantifier scope is not computed during normal reading. Nevertheless, it was possible that NP anaphor reading times would be insensitive to scope processing. Readers might preferentially adopt a singular representation of the indefinite quantifier despite factors affecting scope. Alternatively, readers might establish a co-referential link based on the match between morphological features of the anaphor and its antecedent (e.g., Cloitre \& Bever, 1988), without recourse to representations specifying relative quantifier scope. In either case, we would expect to obtain a processing cost for plural anaphors and no effect of factors affecting relative scope.

Total reading times for Region 3 showed that comprehension difficulty occurred for plurals [plural, $611 \mathrm{msec}$; singulars, $\left.519 \mathrm{msec} ; F_{1}(1,55)=47.47 ; F_{2}(1,47)=30.15\right]$, with no other effects $(F \mathrm{~s}<1.8)$. Evidently, total reading times for NP anaphors were insensitive to factors affecting relative scope, despite evidence for on-line scope processing at Region 2. First-pass times enabled us to determine which factors affected initial processing. Reading times were longer for plurals than for singulars [432 vs. $370 \mathrm{msec} ; F_{1}(1,55)=52.02 ; F_{2}(1,47)=64.09$ ], with no other main effects $\left(F_{\mathrm{S}}<1.7\right)$. The quantifier and grammatical order interaction was unpredicted $\left[F_{1}(1,55)=\right.$ $16.47 ; F_{2}(1,47)=4.87$, with longer DO-first reading times for $a$-every than for every- $a$ [425 vs. $388 \mathrm{msec}$; $\left.F_{1}(1,55)=11.35 ; F_{2}(1,47)=5.05\right]$, but no significant IO-first difference (a-every, $387 \mathrm{msec}$; every-a, $405 \mathrm{msec}$; $\left.F_{\text {s }}<2.8\right)$. Other two-way interactions were not significant $\left(F_{\mathrm{S}}<2.9\right)$. Although there was a three-way interaction $\left[F_{1}(1,55)=4.96 ; F_{2}(1,47)=5.50\right]$, the pattern of means did not match with predictions made by the grammatical hierarchy principle.

Different lexical items were used for NP anaphors when the indefinite quantifier was the first or second quantifier, with anaphors based on the same noun for in- 
definite DOs (e.g., interview) and for indefinite IOs (e.g., reporter). It was possible that the three-way interaction at the NP anaphor was due to interactive influences of content differences across conditions and a preference for singular continuations. Two 2 (grammatical order) $\times 2$ (number) ANOVAs comparing first-pass reading times for anaphors based on the same noun showed that this was the case. Reading times for sentences with indefinite DOs (i.e., items A and D in Table 1) were longer for plurals than for singulars [454 vs. $376 \mathrm{msec}$; $\left.F_{1}(1,55)=40.94 ; F_{2}(1,47)=51.62\right]$, with no other significant effects $\left(F_{\mathrm{S}}<3.3\right)$. Reading times for sentences with indefinite IOs (i.e., items B and C in Table 1) were longer for plurals than for singulars [412 vs. $366 \mathrm{msec}$; $\left.F_{1}(1,55)=20.27 ; F_{2}(1,47)=21.41\right]$, with no other effects $\left(F_{\mathrm{S}}<1\right)$. Thus, first-pass times supported total times in showing a preference for singular anaphors and no effect of factors affecting relative scope.

\section{Region 4}

There were no significant first-pass or total reading time effects at Region $4\left(F_{\mathrm{s}}<4.5\right)$.

\section{DISCUSSION}

Eye tracking enabled us to detect distinct effects at quantified and NP anaphor regions. Off-line judgment data suggested a role for quantifier and grammatical order in scope resolution. Total times at the quantified region showed that these factors interacted during on-line scope computation. Quantifier and grammatical hierarchy principles had a conjoint influence, causing reading difficulty when they conflicted, with longer total reading times for every-a DO-first sentences and for $a$-every IO-first sentences. Thus, the results supported Ioup's (1975) claim for an interaction involving quantifier and grammatical hierarchies during scope ambiguity resolution, with competition between alternative interpretations incurring a processing cost when principles were in conflict. Total times also were longer when $a$ preceded every, consistent with Fodor's (1982) claim that linear order interacts with quantifier characteristics, with readers first representing the indefinite quantifier as a singular and then incurring a cost when revising it to a plural after encountering every. Thus, we obtained novel findings concerning the processing of relative quantifier scope. Our results suggest that factors influencing relative scope can affect the processing of the ambiguity during normal reading. Kurtzman and MacDonald (1993) hypothesized that competition might guide scope ambiguity resolution. Our results provide evidence for such a process. It was surprising to obtain both linear and grammatical order effects. Ioup (1975) had argued that apparent linear order effects in some studies might, instead, be explained by the relative grammatical function of the quantified phrases. One possibility is that whereas the relative grammatical function of quantified phrases provides syntactic cues to relative scope, linear order reflects frequency-based expectations.
First- and second-pass reading times showed that effects for IO-first sentences were delayed and occurred during reinspection of the ambiguity. We attributed the absence of first-pass IO-first effects to an additional syntactic processing load incurred because of their noncanonical construction, causing interpretative processes to be delayed.

Results at the NP anaphor showed a robust effect of number, with shorter total reading times for singular continuations regardless of relative quantifier scope. First-pass effects were attributable both to a preference for singular continuations and to content differences at the NP anaphor region. The results did not support hypotheses predicting that factors influencing relative scope would affect the processing of NP anaphors. However, effects obtained at the quantified region allowed us to discount the possibility that readers compute scope only when the task includes an explicit judgment (Gillen, 1991).

We considered two possible explanations for the absence of scope effects at the NP anaphor. First, readers may have adopted a singular interpretation of the indefinite quantifier regardless of factors affecting relative scope. In this respect, the reading time results were consistent with our judgment task results, with a weak bias for a plural interpretation of the indefinite quantifier when every was likely to take wide scope. Alternatively, readers may have obtained a coherent understanding of the text without interpreting the anaphor with respect to a representation of sentence meaning specifying relative scope. The results of other studies (e.g., Cloitre \& Bever, 1988) suggest that NP anaphors are interpreted with respect to a surface representation of the text, with coreference computed on the basis of the match between morphological features of the anaphor and its antecedent. Our readers may have experienced difficulty in processing plural anaphors when the linguistic form of the antecedent was singular, irrespective of whether it referred to one or many entities. Whichever account is correct, our results suggest that NP anaphor reading times are insensitive to factors affecting the processing of relative scope during normal reading. Other researchers (e.g., Kurtzman \& MacDonald, 1993; Tunstall, 1998) may have obtained effects at continuations because they used tasks that encouraged participants to evaluate possible interpretations of the ambiguity and may not reflect normal reading processes. Nevertheless, we have shown that relative scope is computed during normal reading, with competition between alternative interpretations causing reading difficulty during the processing of the ambiguity.

\section{REFERENCES}

Batyen, R. H., Piepenbrock, R., \& Gulikers, L. (1995). The CELEX lexical database [CD-ROM]. Philadelphia: University of Pennsylvania, Linguistic Data Consortium.

Catlin, J., \& Micham, D. L. (1975). Semantic representations as procedures for verification. Journal of Psycholinguistic Research, $\mathbf{4}_{2}$ 209-225.

Cloitre, M., \& Bever, T. G. (1988). Linguistic anaphors, levels of representation, and discourse. Language \& Cognitive Processes, 3 , 293322. 
Ferreira, F., \& Clifton, C. (1986). The independence of syntactic processing. Journal of Memory \& Language, 25, 348-368.

FoDOR, J. D. (1982). The mental representation of quantifiers. In S. Peters \& E. Saarinen (Eds.), Processes, beliefs, and questions: Essays on formal semantics of natural language and natural language processing (pp. 129-164). Dordrecht: Reidel.

FoDOR, J. D., \& SAG, I. (1982). Referential and quantificational indefinites. Linguistics \& Philosophy, 5, 355-398.

Frazier, L., PACht, J. M., \& RAYNER, K. (1999). Taking on semantic commitments: II. Collective versus distributive readings. Cognition, 70, 87-104.

GILLEN, K. (1991). The comprehension of doubly quantified sentences. Unpublished doctoral dissertation, University of Durham.

Ioup, G. (1975). Some universals for quantifier scope. In J. Kimball (Ed.), Syntax and semantics (Vol. 4, pp. 37-58). New York: Academic Press.

JoHnSON-LAIRD, P. N. (1969). On understanding logically complex sentences. Quarterly Journal of Experimental Psychology, 21, 1-13.

Kurtzman, H. S., \& MacDonald, M. C. (1993). Resolution of quantifier scope ambiguities. Cognition, 48, 243-279.

LAKoFf, G. (1971). On generative semantics. In D. D. Steinberg \& L. A. Jakobovits (Eds.), Semantics: An interdisciplinary reader in philosophy, linguistics and psychology (pp. 232-296). Cambridge: Cambridge University Press.
LARSON, R. (1988). On the double object construction. Linguistic Inquiry, 19, 335-391.

Liversedge, S. P., \& Findlay, J. M. (2000). Saccadic eye movements and cognition. Trends in Cognitive Sciences, 4, 6-14.

Micham, D. L., Catlin, J., VanDerveer, N. J., \& Loveland, K. A. (1980). Lexical and structural cues to quantifier scope relations. Journal of Psycholinguistic Research, 9, 367-377.

PoEsIO, M. (1996). Semantic ambiguity and perceived ambiguity. In K. van Deemter \& S. Peters (Eds.), Semantic ambiguity and underspecification (pp. 159-201). Stanford, CA: CSLI Publications.

RAYNER, K. (1998). Eye movements in reading and information processing: 20 years of research. Psychological Bulletin, 124, 372-422.

Tunstall, S. L. (1998). The interpretation of quantifiers: Semantics and processing. Unpublished doctoral dissertation, University of Massachusetts, Amherst, Department of Linguistics.

van Gompel, R. P. G., Pickering, M. J., \& TraXler, M. J. (2000). Unrestricted race: A new model of syntactic ambiguity resolution. In A. Kennedy, R. Radach, D. Heller, \& J. Pynte (Eds.), Reading as a perceptual process (pp. 621-648). Oxford: Elsevier.

(Manuscript received March 17, 2003; revision accepted for publication December 19, 2003.) 